\title{
The search for Primordial Quark Nuggets among Near Earth Asteroids
}

\author{
J. E. Horvath
}

(C) Springer-Verlag $\bullet \bullet \bullet \bullet$

\begin{abstract}
Primordial Quark Nuggets,remnants of the quark-hadron phase transition, may be hiding most of the baryon number in superdense chunks have been discussed for years always from the theoretical point of view. While they seemed originally fragile at intermediate cosmological temperatures, it became increasingly clear that they may survive due to a variety of effects affecting their evaporation (surface and volume) rates. A search of these objects have never been attempted to elucidate their existence. We discuss in this note how to search directly for cosmological fossil nuggets among the small asteroids approaching the Earth. "Asteroids" with a high visible-to-infrared flux ratio, constant lightcurves and devoid of spectral features are signals of an actual possible nugget nature. A viable search of very definite primordial quark nugget features can be conducted as a spinoff of the ongoing/forthcoming NEAs observation programmes.
\end{abstract}

Keywords Quark Nuggets, Dark matter, Near Earth Asteroids

\section{Introduction}

Primordial quark nuggets (PQNs) have been postulated to contain most of the baryonic number of the universe many years ago (Witten, 1984), being a physical realization of the so-called strange matter hypothesis. The strange matter hypothesis states that a cold, catalyzed form of the quark gluon plasma could be the true ground state of the hadronic matter and, if formed at the QCD scale when color become confined, remnants of

\section{J. E. Horvath}

Instituto de Astronomia, Geofísica e Ciências Atmosféricas, Universidade de São Paulo, Rua do Matão 1226 , 05508-090 São Paulo, SP, Brazil substellar mass could help to "hide" the baryon number content and achieve a large value of $\Omega_{\text {matter }} \sim 0.25$ now favoured by observations. The initial excitement about this possibility gave place to several analysis addressing whether the PQN could survive from the initial hightemperature environment until the lower temperature universe, where the contribution $-T S$ to their free energy make them stable against evaporation-boiling. In fact both processes (evaporation of ordinary hadrons from the surface and bulk conversion -boiling-) have been examined with varying results (Madsen and Olesen, 1991; Alcock and Olinto, 1989; Olesen and Madsen, 1993; Sumiyoshi and Kajino, 1991). Generally speaking, it can be stated that the slower the process, the larger the mass that can survive; thus it is of great interest to pinpoint the realistic models to estimate reliably a surviving mass. It is generally agreed that the latter can not be larger than the mass inside the horizon, corresponding to a baryon number of $A_{\text {hor }}=10^{49}\left(\frac{100 \mathrm{MeV}}{T_{q c d}}\right)$ (where $T_{q c d}$ is the temperature for the confinement phase transition, assumed to be first order); and while no consensus has been achieved on the exact value of this surviving nugget mass, values as low as $A=10^{40}$ have been estimated in the literature (Sumiyoshi and Kajino, 1991; Bhattacharyya et al., 2000). Recently we have shown that the inclusion of pairing effects in strange matter would increase the binding energy of the nugget and hence push down the survival mass substantially (Lugones and Horvath, 2004). There is a strong belief that dense quark matter should undergo pairing of some of all quark flavors, although the precise form of the phase diagram is still under debate (Alford, 2006).

Given this attractive possibility we address here a novel and feasible search for PQN in the asteroidal-mass range; in several senses complementary to the searches of smaller masses (nuclearities) already performed or underway (Finch, 2006). We present a discussion of the 
general features of the nuggets in Section 2. Section 3 addresses the detectability of this population by photometric and spectroscopic techniques. A discussion and some conclusions are summarized in last Section.

\subsection{Primordial Quark Nuggets}

According to the Bodmer-Witten-Terazawa hypothesis (Witten, 1984; see previous work by Bodmer, 1971 and Terazawa, 1979), a stable form of the QGP with high strangeness content could be self-bound and therefore form objects from a minimum (nuclear) size until $\sim M_{\odot}$ at the onset of the general relativistic instability. While the former ("strangelets") are amenable to particle search techniques (Klingenberg, 1999) and the latter may constitute compact stars (Witten, 1984; Alcock, Farhi and Olinto, 1986; Haensel, Zdunik and Schaeffer 1986; Benvenuto and Horvath 1989), in the intermediate range PQNs would resemble extremely compact asteroids. For constant density $\rho=5 \times 10^{14} \mathrm{~g} \mathrm{~cm}^{-3}$ (which is an excellent approximation for this range; see Alcock, Farhi and Olinto, 1986) the size of the nuggets is

$R_{N}=2 \times 10^{2} \times\left[\frac{M_{N}}{10^{-12} M_{\odot}}\right]^{\frac{1}{3}} \mathrm{~cm}$

and we have scaled to the mass corresponding to a baryon number $A=10^{45}$ above the evaporating mass (Lugones and Horvath, 2004).

Because of the large size of the galactic halo, the isotropic nugget flux of this mass-scale is always very small, of the order of $3 \times 10^{-37} \mathrm{~cm}^{-2} \mathrm{~s}^{-1} \mathrm{sr}$ onto the Earth neighborhood. For a minimum approach of one PQN at a distance $D \leq 2 \times$ the Earth-Moon distance, or $2 \times R_{\text {Moon }}$ (see below) a passage would happen at a rate $10^{-6} \mathrm{yr}^{-1}$. This small rate gives no hope for detecting PQNs freely roaming the halo passing by in open orbits.

A captured nugget population in orbit provides much better prospects. To be captured by the gravitational field of the Sun the nuggets coming from an isotropic flux must loose energy and angular momentum. A first viable mechanism would be a number of flyby encounters with stars in the galactic disk. Then a fraction of these slower nuggets, having now $E \gtrsim 0$ respect to the solar potential would be captured in fly-by encounters with Jupiter. Let us stress that even if the distribution of PQNs will not slow down statistically, individual objects from that distribution may be effectively captured by this mechanism. The PQNs with this mass-scale do not slow down appreciably even by passing through stars, since the basic energy loss rate $d E / d t=-\pi R_{N}^{2} \rho_{\text {matter }} v_{N}^{3}$ (with $\rho_{\text {matter }}$ the density of the traversed stellar material and $v_{N}$ the nugget velocity $\sim 250 \mathrm{~km} \mathrm{~s}^{-1}$ ) is many orders of magnitude smaller than the incoming kinetic energy of the nugget.

The estimated number of PQNs effectively captured is always tiny, but perhaps enough to yield $10-100$ of them bound to the solar system out of the $\sim 10^{23}$ existing in the whole galactic population. Note that this capture rate, if extrapolated to all the stars in the disk, would still mean a total capture nugget fraction of $\leq 10^{-10}$. A variety of orbital elements is expected because of the random incidence, however, since the nuggets would have Apollo-like, planet-crossing orbits, their residence in the solar system would be limited to a dynamical time of $\leq 10 \mathrm{Myr}$. Finally, there should be a huge number $\left(\geq 10^{7}\right)$ of PQN at a given time inside the bounds of the solar system, adding up to a mass comparable to several times the Earth's mass. They may be perturbed and directed to the inner solar system much in the same way that ordinary cometary nuclei do, only that they would remain unseen most of the time. The identification of the tiny dense nuggets among the subpopulation of NEAs would be the subject of the next section.

\section{How to detect nuggets among NEAs}

A great deal of attention has been paid recently to the issue of NEAs and the possible hazards for the Earth in the case of direct collisions. Actually, a few observing programs to look for close-by encounters are already operating (for example, Spacewatch, see Mc Millan, 2006) or being implanted. A huge number of small NEAs, those not very hazardous to the Earth in the ballpark of $\sim$ tens of meters are being currently detected. The observations indicate that at a given time $>100$ asteroids pass closer than the Moon, while at least one of $\sim 30 \mathrm{~m}$ and $\simeq 10$ of about $10 \mathrm{~m}$ collide with the Earth each year. At a distance comparable to the Moon these would have magnitudes $m_{v}=13$ and 15.5 respectively (assuming, as usual, a zero phase angle; see Paczyński, 2006). These fast-moving boulders would be visible for $\mathrm{a} \sim$ days at most because of their high velocities relative to the Earth, of the order of $20 \mathrm{~km} \mathrm{~s}^{-1}$.

As is well-known, one of the simplest forms for determining the albedo $A$ (and hence gather information on their composition) of an asteroid is to perform at least simultaneous $V$ and $I$ images to infer $A$ from the quotient of fluxes. The exotic nature of the nuggets allows one relatively easy form of distinguishing them from conventional asteroids: since the strange quark matter is expected to have a plasma frequency as high 
as $20 \mathrm{MeV}$ (well in the hard $\gamma$-ray frequencies), the bare quark surface would act as a perfect mirror to the incident solar light. Hence, contrary to the case of even metallic asteroids for which $A \sim 0.1$, we expect albedos $\approx 1$ and therefore a quotient $F_{V} / F_{I}$ much larger than any reasonable normal surface. Therefore a PQN would appear to have a magnitude $m_{V} \simeq 20$ at a distance $\sim R_{M o o n}$, looking like a larger "normal" asteroid but showing the abnormal flux quotient. Specific programmes like the Spacewatch (Mc Millan, 2006) already mentioned and several other programs (see for example the compilation of the NASA, 2007) currently monitor objects fainter than $m_{V} \sim 20$, and should be able to detect $\sim 1 \mathrm{~m}$ nuggets out to $\sim R_{M o o n}$ (hence the distance estimate given in the former Section). A recent discussion by Paczyński (2006) has shown the convenience of a satellite at the L1 Lagrangian point for the NEAs study and early alert. The main difficulties for such identifications are the high proper motion of the candidates, which render them observable for a short time $\sim 1$ day, and also the large number of candidates in a given night, provided that there is no simple way of selecting a subsample, for instance, based on orbits.

A second prediction for these objects is that their spectra would be essentially solar and devoid of any characteristic line that distinguish asteroidal compositions. This seems promising, although spectroscopy of dim fast-moving objects is certainly difficult. In any case the slower candidates (say, with $\mu \leq 1 " / s$ ) could be scanned by suitably setup devices. Thus both the photometric and spectroscopic measurements would show signatures of the exotic nugget features difficult to miss for good observational data. This feature (and the former flux quotient as also) implicitly assumes that the surface of the nugget is not covered with normal matter, which would restore the ability of radiating conventionally the reflecting light. The situation is quite like the discussion of strange stars (Alcock, Farhi and Olinto, 1986; Haensel, Zdunik and Schaeffer 1986; Benvenuto and Horvath 1989), but now referring to much smaller objects, 12 orders of magnitude less massive than a strange star, with a correspondingly small surface gravity. This consideration supports, but does not prove, the expectation of a bare surface.

A third complementary signature could be obtained by precise stellar occultation observations, given that the tiny nugget with high albedo would mimic a larger radius asteroid (at least 10 times bigger) but would show a very short occultation time corresponding to a small and sharp edge ball. Even the lack of occultation where it should be one (if the asteroid was normal) could be helpful for characterizing the PQN candidates. Finally we note that a very uniform light curve, (in contrast with most of the asteroids of irregular size which tumble and rotate) is expected from the spherical superdense nugget, quite independently of the wavelength. This completes a short list of testable predictions for these objects.

Several contemporary sources (as opposite to the discussed primordial population) that could supply a flux of small nuggets have been advanced, like the disruption of binary strange stars (Madsen, 2005; however see Kluźniak and Lee, 2002 for a calculation of a strange star-black hole encounter not leaving any ejected debris). It is amusing to note that long ago Friedman and Caldwell (1991) predicted the stripping of nuggets of precisely $A=10^{45}$ from the simple balance of tidal and surface forces in a binary merging. However, the small number of events of this type $\sim 10^{-5} \mathrm{yr}^{-1}$ argues against stripped nuggets of this origin being a significant source when compared to the hypothetical primordial population, and in any case there is no way for distinguishing both.

The possibility of a direct impact onto the Earth is extremely small (about one event per Hubble time) for halo PQNs, but grows considerably for a captured population. Specific signatures of such an hypothetic collision (likely giving rise to a huge epilinear earthquake) have never been worked out in full detail. However, lighter PQNs from astrophysical origin may be candidates to direct impacts with masses down to tens of tons (see Herrin, Rosenbaum and Teplitz, 2006, for this milder but related signal search).

\section{Conclusions}

We have discussed some observations which may possibly test the presence of a population of primordial quark nuggets captured in solar orbits. We have been led by the fact that the mass range predicted by theoretical calculations falls precisely in the ballpark of small asteroids, and by the existence of advanced programmes of NEAs search. We suggested a few observational tests to reveal the nature of the nuggets as they approach the Earth. If an efficient setup for the fast-moving candidates can be established it would be possible to identify some definite signatures predicted by the basic physics of the nugget surface reflecting sunlight. Although the search for PQNs could be difficult and take several years, the payoff would be uttermost rewarding even if they are not finally found. In any case, valuable information about the normal asteroid population would be gathered. Few other programmes could be suitable for searching such an elusive primordial relics. 
Acknowledgements $\mathrm{CNPq}$ (Brazil) is acknowledged for financing JEH through a fellowship. C. Beaugé, F. Roig and S. Ferraz-Mello have provided scientific advice to this work. Finally, the criticisms of an anonymous referee have added a few interesting points to the present article. 


\section{References}

Alcock, C., Olinto, A.V.: Phys. Rev. D 39, 1233 (1989)

Alcock, C., Farhi, E., Olinto, A.V.: ApJ310, 261 (1986)

Alford, M.G.: hep-lat/0610046 (2006) and references therein

Benvenuto, O.G., Horvath, J.E.: MNRAS241, 43 (1989)

Bhattacharyya, A., Jan-e Alam, Sarkar, S., Roy, P., Sinha, B., Raha, S., Bhattacharjee, P.: Phys. Rev. D61, 083509 (2000)

Bodmer A.: Phys. Rev. D4, 1601 (1971)

Caldwell, R.R., Friedman, J.L.: Phys. Lett. B264, 143 (1991)

Finch, E.: nucl-ex/0605010 (2006)

Haensel, P.; Zdunik, J. L.; Schaefer, R.: A\&A160, 121 (1986)

Herrin, E. T.; Rosenbaum, D. C., Teplitz, V. L.: Phys. Rev. D 73, 043511 (2006)

Klinbenerg, R.: Jour. Phys. G25, R273 (1999)

Kluźniak, W., Lee, W.: MNRAS335, L29 (2002)

Lugones, G., Horvath, J.E.: Phys. Rev. D69, 063509 (2004)

Madsen, J., Olesen, M.: Phys. Rev. D 43, 1069 (1991)

Madsen, J.: J. Phys. G31, 833 (2005)

McMillan, R. S., in Near Earth Objects, Our Celestial Neighbors: Opportunity and Risk, International Astronomical Union. Symposium 236, (2006), unpublished

NASA info available at http://neo.jpl.nasa.gov/programs/ (2007)

Olesen, M., Madsen, J.: Phys. Rev. D47, 2313 (1993)

Paczyński, B.: astro-ph/0609161 (2006)

Sumiyoshi, K., Kajino, T.: Nucl. Phys. B. Proc. Supp. 24, 80 (1991)

Terazawa H.: INS-Report 336 (1979), unpublished

Witten E.: Phys. Rev. D 30, 272 (1984) 